\title{
Fecundidad, volumen del huevo y rendimiento reproductivo de cinco especies de porcelánidos intermareales del norte de Chile (Decapoda, Porcellanidae)
}

\author{
Patricio Hernáez \& Sergio Palma \\ Escuela de Ciencias del Mar, Pontificia Universidad Católica de Valparaíso, Casilla 1020, \\ Valparaíso, Chile \\ E-mail: phernaez@eudoramail.com, spalma@ucv.cl
}

\begin{abstract}
RESUMEN. Se analiza la fecundidad, rendimiento reproductivo y volumen del huevo de 286 hembras ovígeras de crustáceos porcelánidos pertenecientes a Petrolisthes granulosus, P. tuberculatus, Allopetrolisthes angulosus, A. spinifrons y Liopetrolisthes patagonicus, colectadas al sur de Iquique. El número de huevos se correlacionó positivamente con la talla de las hembras mostrando un buen ajuste a la función de poder. Las hembras de A. spinifrons y $L$. patagonicus presentaron las mayores cantidades de huevos, con $612 \pm 437$ y $413 \pm 284$ huevos respectivamente. $P$. granulosus fue la especie más pequeña $(7,3 \pm 0,80 \mathrm{~mm} \mathrm{Lc})$, pero se caracterizó por incubar huevos de gran tamaño $\left(0,184 \pm 0,038 \mathrm{~mm}^{3}\right)$. La pérdida potencial de huevos durante el desarrollo embrionario no fue significativa $(\mathrm{p}>0,05)$ en ninguna de las especies analizadas. Durante el periodo de incubación del embrión, el tamaño del huevo aumentó entre un $43 \%$ (P. granulosus) y un $88 \%$ (L. patagonicus). Este aumento fue más importante en las especies sometidas a menor presión de desecación, lo cual se relacionaría con la distribución vertical de cada especie en la zona intermareal. La inversión de energía en la reproducción fluctuó entre 3,7 y 7,4\%, valores que están dentro del rango descrito para otros crustáceos decápodos.
\end{abstract}

Palabras claves: fecundidad, rendimiento reproductivo, volumen del huevo, porcelánidos, Chile.

\section{Fecundity, egg volume and reproductive output of five species of intertidal porcellanids from northern Chile (Decapoda, Porcellanidae)}

\begin{abstract}
The fecundity, egg volume and output reproductive of 286 ovigerous females belonging to Petrolisthes tuberculatus, $P$. granulosus, Allopetrolisthes angulosus, A. spinifrons and Liopetrolisthes patagonicus, collected in the rocky intertidal of northern Chile in December of 1999, was analyzed. In each species, the egg number was positively correlated with female size showing a good fit to the power function. Females of A. spinifrons and L. patagonicus were the species that produced the highest egg number $(612 \pm 437$ and $413 \pm 284$ eggs, respectively). The females of $P$. granulosus incubated eggs of great size $\left(0.184 \pm 0.038 \mathrm{~mm}^{3}\right)$, and were smaller in corporal size $(7.3 \pm 0.80 \mathrm{~mm} \mathrm{Lc})$ than the rest of the analyzed species. The potential loss of eggs during the developmental stages it is not significative ( $>0.5$ ). During the incubation period, the egg size increased between $43 \%$ (P. granulosus) and $88 \%$ (L. patagonicus). This phenomenon was more important in the populations subjected to less drying pressure, which would be related to the vertical distribution of species in the intertidal area. The reproductive output fluctuated between 3.7 and $7.4 \%$, values within the range described for other porcellanids.
\end{abstract}

Key words: fecundity, egg volume, reproductive output, porcellanids, Chile.

\section{INTRODUCCIÓN}

Los crustáceos decápodos han desarrollado diferentes estrategias de supervivencia que han permitido su éxito en una gran variedad de hábitats. Esta característica hace que estos organismos sean bastan- te interesantes, especialmente con relación a sus aspectos reproductivos (Pinheiro \& Fransozo, 1995). En este sentido, diversos aspectos de la biología reproductiva como la producción de huevos y la 
estimación de fecundidad son fundamentales para comprender y asegurar la renovación natural de sus poblaciones, como también para efectuar un manejo adecuado de las pesquerías en aquellas especies de importancia comercial (Caddy, 1989)

En los crustáceos la fecundidad permite estimar el estado reproductivo en que se encuentra la población y generalmente, se ha definido como el número de huevos liberados por una hembra en un solo proceso de desove o durante un periodo determinado de su ciclo de vida (Swartz, 1978; Jones \& Simons, 1983; Palma \& Arana, 1997; Hernáez, 2001) o como el número de huevos contenidos bajo el abdomen de las hembras (Stechey \& Somers, 1995). En los decápodos, la fecundidad varía bastante de una familia a otra y se pueden contar desde cientos de huevos en algunos Porcellanidae (Antezana et al., 1965; Lardies \& Wehrtmann, 1996; Hernáez \& Pinheiro, 2001), hasta varios miles en algunos Galatheidae (Palma \& Arana, 1997), Cancridae (Hines, 1991) o Xanthidae (Antezana et al., 1965). Siempre es conveniente tener en cuenta las diferentes perspectivas de la fecundidad. En la mayoría de los decápodos la hembra porta los huevos bajo el abdomen durante su desarrollo y esto permite estimar no solo la fecundidad, sino que además, estudiar las características del huevo durante el desarrollo embrionario.

El volumen del huevo está considerado como un indicador del contenido de energía para el desarrollo del embrión (Herring, 1974; Levitan, 1996). Con frecuencia este hecho se relaciona con el tipo de estrategia que las hembras adoptan en respuesta a determinadas condiciones del ambiente, como temperatura y fotoperíodo (Clarke, 1992). Por lo tanto, la estimación del volumen del huevo es importante para comprender los mecanismos que la población emplea para adaptarse al medio ambiente y sobrevivir.

Durante el desarrollo, el huevo aumenta de volumen debido al crecimiento del embrión. Lardies $\&$ Wehrtmann (1996) mencionan que este crecimiento es controlado por el grosor y resistencia de la membrana, que se opone al aumento desmesurado del volumen del huevo. Esto revela la importancia de la membrana en la sobrevivencia del embrión, sobre todo en aquellas especies ubicadas en la parte superior del intermareal que están sometidas a una mayor presión de desecación, ya que la membrana puede ser uno de los factores que regula el balance entre los fluidos internos y el medio externo.
En la zona intermareal y submareal de la costa americana, la familia Porcellanidae es una de las más representativas (Haig, 1960; Carvacho, 1980). Sus componentes desempeñan un papel importante en la recuperación de la materia orgánica (Viviani, 1969; Sanhueza et al., 1975) y en algunos casos, se han utilizado para indicar el grado de diversidad ecológica en determinados ambientes litorales (Werding, 1984). En Chile, esta familia está constituida por 16 especies pertenecientes a los géneros Pachycheles, Megalobrachium, Petrolisthes, Allopetrolisthes y Liopetrolisthes (Haig, 1955; Carvacho, 1980; Lardies \& Wehrtmann, 1996). Estos crustáceos se caracterizan por un desarrollo embrionario abreviado, compuesto por tres estados larvales, dos zoeas y una megalopa, cuyos máximos de abundancia en aguas templadas de la costa chilena ocurren en primavera y verano (Palma, 1980; Ulloa \& Palma, 1998).

En este trabajo se presentan y discuten algunos aspectos de la biología reproductiva, como la fecundidad, rendimiento reproductivo y volumen del huevo, de cinco especies de porcelánidos litorales, cuyo conocimiento es muy limitado en la zona norte de Chile y se compara con la biología de otras especies colectadas en otras zonas de la costa chilena.

\section{MATERIALES Y MÉTODOS}

Se analizaron 286 hembras ovígeras distribuidas en 11 ejemplares de Petrolisthes tuberculatus (Guérin, 1837), 80 de $P$. granulosus (Guérin, 1835), 79 de Allopetrolisthes angulosus (Guérin, 1835), $59 \mathrm{de} A$. spinifrons (H. Milne Edwards, 1837) y 57 de Liopetrolisthes patagonicus (Cunningham, 1871). Todas las hembras se colectaron en el intermareal rocoso del sector denominado Tres Islas $\left(20^{\circ} 18^{\prime} 55^{\prime \prime} \mathrm{S}, 70^{\circ} 08^{\prime} 17^{\prime \prime} \mathrm{W}\right)$, ubicado $7 \mathrm{~km}$ al sur de Iquique (Fig. 1). El sector analizado tenía escasa pendiente y gran cantidad de piedras pequeñas, bajo las cuales se refugiaban cangrejos porcelánidos y otros invertebrados, como ofiúridos, equinodermos y asteroídeos. Las hembras ovígeras se obtuvieron durante un corto periodo de tiempo, entre el 21 y 29 de diciembre de 1999, para evitar cambios temporales en las características reproductivas de las diferentes especies (Díaz, 1980). Todas las hembras capturadas se almacenaron individualmente para evitar la pérdida de huevos y apéndices, y se trasladaron al laboratorio donde fueron congeladas para su posterior análisis. 


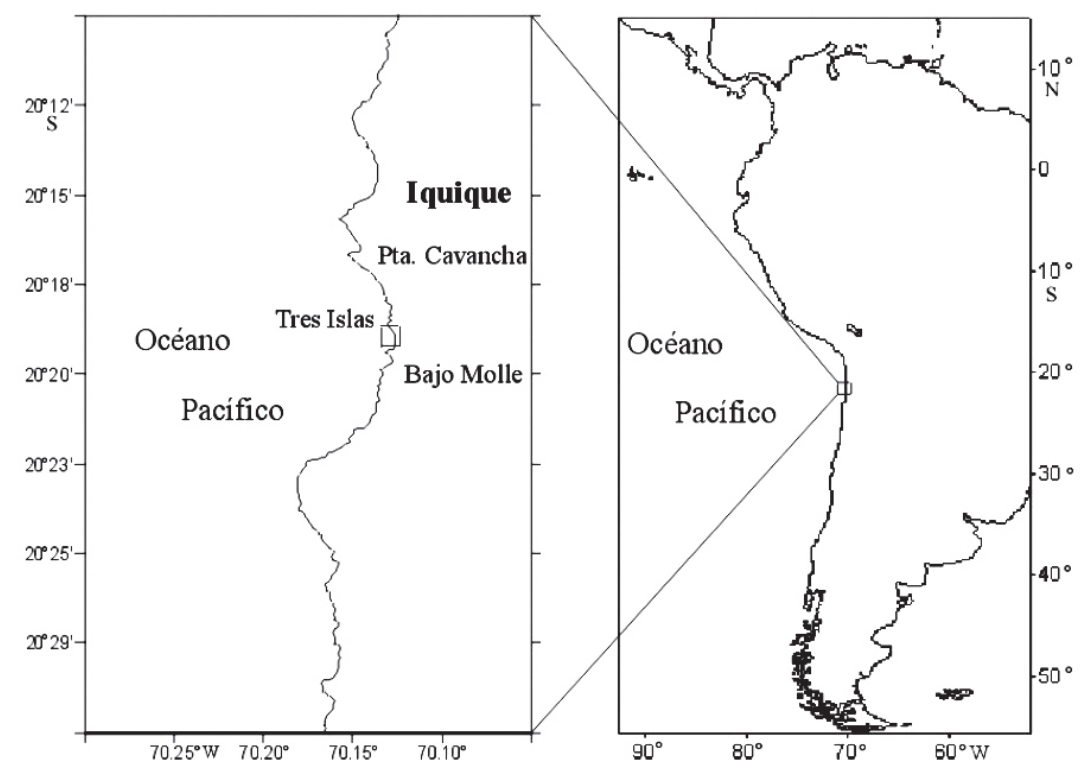

Figura 1. Ubicación geográfica del área de estudio.

Figure 1. Geographic location of study area.

Las hembras ovígeras se identificaron considerando los trabajos de Haig $(1955,1960)$ y Guzmán (1999). En cada individuo se midió la distancia entre el ápice del rostro y el margen posterior del caparazón (Lc), usando un pie de metro de $0,05 \mathrm{~mm}$ de precisión. De acuerdo a la distribución por rangos de $0,1 \mathrm{~mm}$ de Lc de las hembras ovígeras, se determinó la talla mediana de portación para cada especie.

Para determinar el número de huevos (Nh) se removió la masa ovígera desde los pleópodos de la hembra, y los huevos se contaron y clasificaron según su estado de desarrollo embrionario. Debido a que la pérdida potencial de huevos durante la incubación puede ser considerable en algunas especies, se aplicó un análisis de covarianza (ANCOVA, $\mathrm{p}<0,05)$ a la relación entre el número de huevos y la longitud del caparazón en cada estado, para descartar la posible influencia del estado de desarrollo en el número de huevos portados. Cuando la relación entre estados fue significativa $(\mathrm{p}<0,05)$ se aplicó el test a posteriori de Tukey para establecer la semejanza entre cada par de regresión (Sokal \& Rohlf, 1981).

El estado de desarrollo de los huevos (E) se determinó considerando la forma del huevo y los criterios utilizados para Petrolisthes laevigatus (Lardies \& Wehrtmann, 1996). Consecuentemente, los hue- vos se clasificaron en Estado I (E-I): huevo redondeado, vitelo uniforme y sin mancha ocular visible; Estado II (E-II): huevo ovoide, mancha ocular alargada y escasamente visible; y Estado III (E-III): huevo ovoide, ojos visibles bien desarrollados e intensamente pigmentados y abdomen libre.

El volumen del huevo ( $\mathrm{Vh}$ ) se calculó separando arbitrariamente 20 unidades por hembra y se midió el diámetro mayor (a) y menor (b) de cada huevo con un microscopio con ocular graduado, para lo cual se descartaron los huevos que presentaron algún grado de deterioro debido al periodo de congelación. Los datos obtenidos se incorporaron en la fórmula $\mathrm{Vh}=1 / 6\left(\mathrm{a} \cdot \mathrm{b}^{2} \cdot \pi\right)$ propuesta por Corey $\&$ Reid (1991). El rendimiento reproductivo (Ro) se estimó mediante la ecuación señalada por Clarke $e t$ al. (1991), donde Ro = peso seco de la masa ovífera (Wo)/ peso seco de la hembra sin huevos (Wh), siguiendo la metodología utilizada para $P$. laevigatus (Lardies \& Wehrtmann, 1996) y considerando solo las hembras con huevos en estado inicial de desarrollo (E-I).

Posteriormente, se efectuó un análisis de regresión entre el número de huevos y la longitud del caparazón en cada especie. Este análisis se aplicó a los datos empíricos y también a los puntos medios de cada rango de talla de $0,1 \mathrm{~mm}$, para demostrar que el ajuste de los puntos medios disminuye la 
varianza de los datos en cada intervalo de talla. Para representar la fecundidad de cada especie se seleccionó el modelo potencial $\left(\mathrm{y}=\mathrm{a} \cdot \mathrm{x}^{\mathrm{b}}\right)$, que ha sido utilizado para numerosos crustáceos (Hines, 1988, 1991; Reid \& Corey, 1991; Pinheiro \& Fransozo, 1995; Palma \& Arana, 1997). Para determinar la relación entre la inversión de energía en la reproducción y el tamaño de las hembras, el rendimiento reproductivo (Ro) de cada especie se correlacionó linealmente con la talla corporal de las hembras en E-I, tomando como variable dependiente Ro. Luego, mediante el test de Tukey se compararon entre sí las medias de cada una de las variables medidas para establecer posibles diferencias interespecíficas.

\section{RESULTADOS}

En la mayoría de los casos, las especies analizadas presentaron un patrón de zonación determinado, cuyo ordenamiento desde la zona intermareal superior a la inferior fue $P$. granulosus, A. angulosus, $A$. spinifrons asociado a $L$. patagonicus y $P$. tuberculatus. En esta zonación llamó la atención la ausencia de $P$. violaceus (Guerín, 1831), que es un componente común en el sistema litoral chileno (Antezana et al., 1965).

Las tres variables calculadas, longitud del caparazón, número de huevos portados y volumen del huevo presentaron diferencias significativas $(\mathrm{p}<0,001)$ entre las diferentes especies analizadas (Tabla 1). Para determinar si estas diferencias ocurrieron entre todas las especies o solo entre aquellas del mismo género, se aplicó un test a posteriori que mostró que el promedio de talla de las hembras de un mismo género, presentó diferencias significativas (Tabla 1; Tukey, $\mathrm{p}<0,05$ ), separándose $P$. granulosus y A. spinifrons, del gupo formado por $P$. tuberculatus, A. angulosus y L. patagonicus. En cambio, según la cantidad de huevos portados, solo las hembras de Allopetrolisthes presentaron diferencias significativas al interior del mismo género (Tabla 1; Tukey, $\mathrm{p}>0,05$ ).

De acuerdo al tamaño corporal, las hembras más pequeñas correspondieron a $P$. granulosus, con un promedio de 7,3 $\mathrm{mm}$ de Lc y un escaso número de huevos (115 huevos), pero de gran tamaño $(0,184$ $\left.\mathrm{mm}^{3}\right)$. Las hembras más grandes pertenecieron a $A$. spinifrons, con un promedio de 12,6 mm de Lc y una elevada cantidad de huevos (612 huevos), relativamente grandes $\left(0,127 \mathrm{~mm}^{3}\right)$ (Tabla 1$)$. La talla mediana de portación fue de 7,2 $\mathrm{mm}$ en $P$. granulosus, 9,5 $\mathrm{mm}$ en $P$. tuberculatus, 9,8 $\mathrm{mm}$ en A. angulosus, 13,0 mm en $A$. spinifrons y $11,3 \mathrm{~mm}$ en L. patagonicus.

Todas las hembras presentaron un incremento de la fecundidad con respecto a su talla corporal (Tabla 2). En casi todas las especies, el número de huevos por talla no varió significativamente $(p>0,05)$

Tabla 1. Rango y promedio de la longitud del caparazón (Lc), número de huevos producidos (Nh) y volumen del huevo en cinco especies de porcelánidos. N: número de individuos, DE: desviación estándar. La comparación de medias entre las distintas variables seguidas por una misma letra en cada columna, indica que no difieren estadísticamente entre cada especie $(\mathbf{p}>0,05)$.

Table 1. Range and average of the carapace length (Lc), number of produced eggs (Nh) and egg volume in five species of porcellanids. N: number of individuals, DE: standard deviation. The mean comparison between the different variables followed by a same letter in each column, indicates that they are not statistically different in each species ( $\mathbf{p}>0,05)$.

\begin{tabular}{|c|c|c|c|c|c|c|c|c|c|c|}
\hline \multirow[t]{2}{*}{ Especie } & \multicolumn{3}{|c|}{$\begin{array}{l}\text { Longitud caparazón } \\
(\mathbf{m m})\end{array}$} & \multirow[b]{2}{*}{ DE } & \multicolumn{3}{|c|}{$\begin{array}{c}\text { Número de huevos } \\
\text { (unidades) }\end{array}$} & \multicolumn{3}{|c|}{$\begin{array}{c}\text { Volumen huevo } \\
\left(\mathrm{mm}^{3}\right)\end{array}$} \\
\hline & $\mathbf{N}$ & Rango & Media & & Rango & Media & DE & Rango & Media & DE \\
\hline P. tuberculatus & 11 & $8,8-13,5$ & $10,2 \mathrm{a}$ & 1,55 & $27-537$ & $213 \mathrm{ab}$ & 156 & $0,079-0,195$ & 0,118 acd & 0,040 \\
\hline P. granulosus & 80 & $4,7-9,0$ & $7,3 \mathrm{~b}$ & 0,80 & $40-225$ & $115 \mathrm{~b}$ & 41 & $0,096-0,265$ & $0,184 \mathrm{~b}$ & 0,038 \\
\hline A. angulosus & 79 & $6,0-16,8$ & $10,1 \mathrm{a}$ & 1,78 & $6-1301$ & $242 \mathrm{a}$ & 249 & $0,054-0,192$ & $0,101 \mathrm{c}$ & 0,036 \\
\hline A. spinifrons & 59 & $7,6-16,3$ & $12,6 \mathrm{c}$ & 2,24 & $33-2138$ & $612 c$ & 437 & $0,070-0,251$ & $0,127 \mathrm{~d}$ & 0,042 \\
\hline L. patagonicus & 57 & $7,0-15,0$ & $10,8 \mathrm{a}$ & 1,79 & $34-1247$ & $413 \mathrm{a}$ & 284 & $0,066-0,201$ & 0,111 acd & 0,037 \\
\hline $\mathrm{F}$ & \multicolumn{4}{|c|}{92,76} & \multicolumn{3}{|c|}{32,11} & \multicolumn{3}{|c|}{56,05} \\
\hline $\mathrm{P}$ & \multicolumn{4}{|c|}{$<0,001$} & \multicolumn{3}{|c|}{$<0,001$} & \multicolumn{3}{|c|}{$<0,001$} \\
\hline
\end{tabular}


entre los diferentes estados de desarrollo (ANCOVA: $P$. tuberculatus, $\mathrm{F}=1,27 ;$. granulosus, $\mathrm{F}=0,52 ;$ A. angulosus, $\mathrm{F}=1,24 ;$ A. spinifrons, $\mathrm{F}=1,93$ ). Solo L. patagonicus presentó diferencias significativas (ANCOVA, $F=4,38 ; p<0,05$ ), recayendo esta diferencia en la producción de huevos en estado inicial de desarrollo (E-I) respecto a los demás estados embrionarios (Tukey, $\mathrm{p}<0,05$ ), cuando las hembras perdieron alrededor de un $34,6 \%$ de los huevos producidos inicialmente. El modelo potencial seleccionado para la relación Nh y Lc describió significativamente la producción de huevos en las diferentes especies de porcelánidos ( $\mathrm{p}<0,05)$, aunque el ajuste entre ambas variables no registró valores altos del coeficiente de determinación (Tabla 3), lo que podría estar asociado a la dispersión de los datos em-

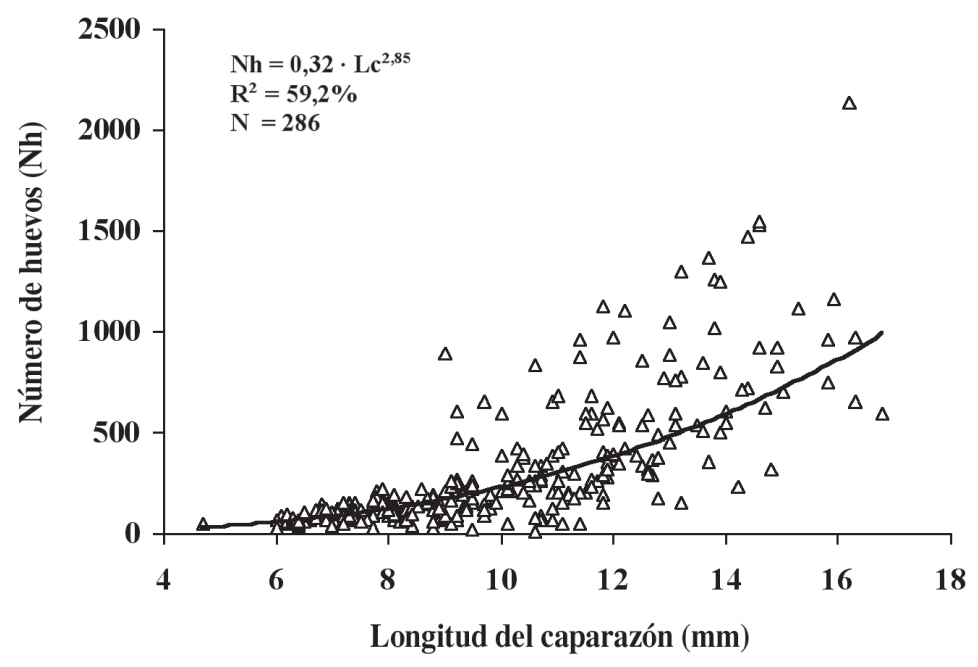

Figura 2. Relación entre el número de huevos portados y la longitud del caparazón, en cinco especies de porcelánidos.

Figure 2. Relationship between the number of eggs and carapace length in five species of porcellanids.

Tabla 2. Fecundidad por clase de talla en cinco especies de porcelánidos. Entre paréntesis se indica el número de individuos utilizados para el cálculo de la media en cada clase de talla.

Table 2. Fecundity by size class in five species of porcellanids. In parentheses the number of individuals used for the calculation of the mean of each size class are indicated.

\begin{tabular}{|c|c|c|c|c|c|}
\hline $\begin{array}{c}\text { Clases de talla } \\
(\mathbf{m m})\end{array}$ & $\begin{array}{l}\text { Petrolisthes } \\
\text { tuberculatus }\end{array}$ & $\begin{array}{r}\text { Petrolisthes } \\
\text { granulosus }\end{array}$ & $\begin{array}{c}\text { Allopetrolisthes } \\
\text { angulosus }\end{array}$ & $\begin{array}{c}\text { Allopetrolisthes } \\
\text { spinifrons }\end{array}$ & $\begin{array}{c}\text { Liopetrolisthes } \\
\text { patagonicus }\end{array}$ \\
\hline $4-5$ & & $45 \pm 0(1)$ & & & \\
\hline $5-6$ & & $66 \pm 0(1)$ & $31 \pm 0(1)$ & & \\
\hline $6-7$ & & $85 \pm 27(29)$ & $65 \pm 0(1)$ & & $34 \pm 0(1)$ \\
\hline $7-8$ & & $131 \pm 29(38)$ & $82 \pm 14(5)$ & $49 \pm 23(2)$ & $110 \pm 82(4)$ \\
\hline $8-9$ & $56 \pm 26$ & $151 \pm 45(11)$ & $106 \pm 40(12)$ & $123 \pm 53(3)$ & $285 \pm 344(5)$ \\
\hline $9-10$ & $181 \pm 74(4)$ & & $173 \pm 92(22)$ & $269 \pm 116(3)$ & $360 \pm 216(9)$ \\
\hline $10-11$ & $292 \pm 124(2)$ & & $217 \pm 151(20)$ & $336 \pm 173(6)$ & $391 \pm 197(8)$ \\
\hline $11-12$ & & & $399 \pm 343(10)$ & $327 \pm 163$ & $436 \pm 258(18)$ \\
\hline $12-13$ & $333 \pm 0(1)$ & & $385 \pm 115$ & $632 \pm 270(8)$ & $525 \pm 290(8)$ \\
\hline $13-14$ & $537 \pm 0(1)$ & & $949 \pm 498$ & $729 \pm 359(12)$ & $847 \pm 363$ \\
\hline $14-15$ & & & & $893 \pm 457(11)$ & $701 \pm 0$ \\
\hline $15-16$ & & & $1165 \pm 0(1)$ & $941 \pm 184$ & \\
\hline $16-17$ & & & $593 \pm 0(1)$ & $1256 \pm 780$ & \\
\hline Total & $213 \pm 156(11)$ & $115 \pm 41(80)$ & $242 \pm 249$ (79) & $612 \pm 437(59)$ & $413 \pm 284(57)$ \\
\hline
\end{tabular}


Tabla 3. Ecuación de regresión para la relación entre el número total de huevos (Nh) y la longitud del caparazón (Lc) en cinco especies de porcelánidos. $\mathrm{N}$ : número de hembras, $\mathbf{R}^{2}$ : coeficiente de determinación, $\mathrm{F}$ : valor calculado de $\mathbf{F}$.

Table 3. Regression equation for the relationships between the total number of eggs (Nh) and the carapace length (Lc) in five species of porcellanids. N: number of females, $R^{2}$ : determination coefficient, F: calculated value of $\mathbf{F}$.

\begin{tabular}{|llllrr|}
\hline \multicolumn{1}{|c}{ Especie } & $\mathbf{X}$ & Función & $\mathbf{N}$ & $\mathbf{R}^{2}$ & F \\
\hline Petrolisthes tuberculatus & $\mathrm{Lc}$ & $\mathrm{Nh}=1,28 \cdot 10^{-3} \cdot \mathrm{Lc}^{5,08}$ & 11 & 0,63 & $15,29 *$ \\
Petrolisthes granulosus & $\mathrm{Lc}$ & $\mathrm{Nh}=0,62 \cdot \mathrm{Lc}^{2,60}$ & 80 & 0,59 & $113,94^{*}$ \\
Allopetrolisthes angulosus & $\mathrm{Lc}$ & $\mathrm{Nh}=0,16 \cdot \mathrm{Lc}^{3,16}$ & 79 & 0,34 & $39,99 *$ \\
Allopetrolisthes spinifrons & $\mathrm{Lc}$ & $\mathrm{Nh}=0,05 \cdot \mathrm{Lc}^{3,61}$ & 59 & 0,65 & $106,39 *$ \\
Liopetrolisthes patagonicus & $\mathrm{Lc}$ & $\mathrm{Nh}=0,21 \cdot \mathrm{Lc}^{3,10}$ & 57 & 0,46 & $46,04 *$ \\
\hline
\end{tabular}

* Valores significativos a nivel de $\mathrm{p}<0,05$

píricos observada en las hembras de mayores tamaños, superiores a $10 \mathrm{~mm}$ de Lc (Fig. 2, Tabla 2).

El volumen del huevo mostró diferencias notorias a lo largo del proceso de incubación. En $P$. tuberculatus y L. patagonicus el volumen de los embriones a punto de eclosionar (E-III) fue un $80 \%$ mayor respecto a su volumen inicial (E-I). Se encontró una relación inversa entre el incremento de volumen entre estados de desarrollo y el patrón de zonación de cada especie. Así las especies distribuidas en la parte superior del intermareal, más alejadas de la línea de marea y que soportan una mayor presión de desecación, como $P$. granulosus y $A$. angulosus, presentaron un incremento en el volumen del huevo mucho menor que el estimado para A. spinifrons, L. patagonicus y P. tuberculatus (Tabla 4).

El volumen del huevo en estado inicial de desarrollo (E-I), fluctuó entre 0,083 y $0,155 \mathrm{~mm}^{3}$ en $A$. angulosus y $P$. granulosus respectivamente (Tabla 4). Entre los distintos estados de desarrollo se determinaron diferencias significativas del volumen del huevo por especie, las cuales fueron más elevadas en los primeros estados de desarrollo (E-I y EII), cuando el huevo mantiene su forma esférica (Tabla 4), mientras que por género no presentó ninguna tendencia (Tukey, $\mathrm{p}<0,05$ ). El peso seco de cada huevo presentó una alta correlación con el volumen del huevo en cada estado de desarrollo, encontrándose que todos los valores de $\mathrm{R}$ fueron significativos a nivel de $\mathrm{p}<0,05$ (E-I, $\mathrm{R}=0,96, \mathrm{~F}=34,84, \mathrm{~N}=$ 129; E-II, $\mathrm{R}=0,95, \mathrm{~F}=29,81, \mathrm{~N}=77$; E-III, $\mathrm{R}=$ $0,88, F=10,31, N=80$ ). Esto demuestra que el embrión aumenta progresivamente de peso a medida que va completando el desarrollo de sus estruc- turas corporales. A excepción de $P$. tuberculatus, las especies restantes presentaron un incremento en el peso seco del huevo durante el desarrollo embrionario, que fluctuó entre un 4,9\% en $A$. angulosus y un $35,3 \%$ en A. spinifrons (Tabla 5). Esta amplia variabilidad también se observó en el peso seco de las hembras, donde en algunas especies como $A$. angulosus y $P$. tuberculatus se estimó una pérdida de un 3,2 y 14,0\% respectivamente, mientras que las demás aumentaron su peso durante la incubación, alcanzando al 44,6\% en A. spinifrons (Tabla 5).

El rendimiento reproductivo de las hembras en estado inicial del desarrollo (E-I), fluctuó entre 3,7\% (A. angulosus) y 7,4\% (L. patagonicus) de su peso seco (Fig. 3), mostrando diferencias significativas entre especies (ANOVA, $\mathrm{F}=4,77 ; \mathrm{p}<0,001 ; \mathrm{N}=$ 129). No se encontró ninguna tendencia en la inversión reproductiva que se relacionara positivamente con el tamaño de las hembras $(\mathrm{R}=0,06 ; \mathrm{F}=0,57)$. Entre las especies del mismo género, el Ro no presentó diferencias significativas (Tukey, $\mathrm{p}>0,05$ ). Además, la relación del Ro con las variables Nh y Wo fue positiva, mostrando una correlación lineal moderada cuando los datos se ajustaron a nivel de $\mathrm{p}<0,001(\mathrm{R}=0,47 ; \mathrm{F}=34,59 ; \mathrm{N}=129 ; \mathrm{R}=0,63 ; \mathrm{F}$ $=81,20 ; \mathrm{N}=129$ respectivamente).

\section{DISCUSIÓN}

La madurez sexual en los decápodos está condicionada principalmente por la temperatura y el fotoperíodo (Kinne, 1970; Annala et al., 1980). A menudo estos factores son responsables de iniciar el proceso de maduración ovárica y testicular, según las características del medio donde se desarro- 
Tabla 4. Volumen del huevo (Vh) durante el periodo de incubación en cinco especies de porcelánidos. N: Número de individuos por estado de desarrollo embrionario, I-III: incremento de volumen durante la embriogénesis. Table 4. Egg volume ( $\mathrm{Vh})$ during the incubation period in five species of porcellanids. N: number of females by developmental stage, I-III: volume increase during the embryogenesis.

\begin{tabular}{|c|c|c|c|c|c|c|c|}
\hline \multirow[t]{3}{*}{ Especie } & \multicolumn{6}{|c|}{ Estado de desarrollo } & \multirow{3}{*}{ I-III (\%) } \\
\hline & \multicolumn{2}{|r|}{ E-I } & \multicolumn{2}{|c|}{ E-II } & \multicolumn{2}{|c|}{ E-III } & \\
\hline & $\mathbf{N}$ & Vh $\left(\mathbf{m m}^{3}\right)$ & $\mathbf{N}$ & Vh $\left(\mathbf{m m}^{3}\right)$ & $\mathbf{N}$ & Vh $\left(\mathbf{m m}^{3}\right)$ & \\
\hline Petrolisthes tuberculatus & 8 & 0,103 & 1 & 0,103 & 2 & 0,189 & 83 \\
\hline Petrolisthes granulosus & 31 & 0,155 & 29 & 0,155 & 20 & 0,222 & 43 \\
\hline Allopetrolisthes angulosus & 36 & 0,083 & 18 & 0,083 & 25 & 0,132 & 59 \\
\hline Allopetrolisthes spinifrons & 25 & 0,104 & 15 & 0,104 & 19 & 0,173 & 66 \\
\hline Liopetrolisthes patagonicus & 29 & 0,085 & 14 & 0,085 & 14 & 0,160 & 88 \\
\hline $\mathrm{F}$ & & 46,48 & & 46,48 & & 24,81 & \\
\hline $\mathrm{P}$ & & $<0,001$ & & $<0,001$ & & $<0,001$ & \\
\hline
\end{tabular}

Tabla 5. Peso seco del huevo y de las hembras ovígeras en estado inicial y final del desarrollo embrionario. Entre paréntesis se indica la pérdida en peso entre ambos estados de desarrollo.

Table 5. Dry weight of the eggs and of the ovigerous females in the initial and final developmental stages. Loss percentages in dry weight between both developmental stages are indicated in parentheses.

\begin{tabular}{|lccccccc|}
\hline \multirow{2}{*}{ Especie } & \multicolumn{3}{c}{ Huevo por estado } & \multicolumn{3}{c|}{ Hembra por estado } \\
\cline { 2 - 8 } & $\mathbf{E}-\mathbf{I}$ & $\mathbf{E - I I I}$ & Diferencia & E-I & \multicolumn{2}{c|}{ E-III } & Diferencia \\
\cline { 2 - 8 } & $\boldsymbol{\mu} \mathbf{g}$ & $\boldsymbol{\mu} \mathbf{g}$ & $\mathbf{( \% )}$ & $\mathbf{g}$ & $\mathbf{g}$ & $\mathbf{( \% )}$ \\
\hline Petrolisthes tuberculatus & 29,36 & 28,02 & $(4,8)$ & 0,1984 & 0,1706 & $(14,0)$ \\
Petrolisthes granulosus & 45,42 & 51,24 & 12,8 & 0,0877 & 0,0968 & 10,4 \\
Allopetrolisthes angulosus & 22,09 & 23,18 & 4,9 & 0,2157 & 0,2088 & $(3,2)$ \\
Allopetrolisthes spinifrons & 23,65 & 32,00 & 35,3 & 0,3947 & 0,5708 & 44,6 \\
Liopetrolisthes patagonicus & 24,40 & 27,16 & 11,3 & 0,1858 & 0,1866 & 0,4 \\
\hline F & 45,00 & 34,66 & & 45,74 & 23,01 & \\
P & $<0,001$ & $<0,001$ & & $<0,001$ & $<0,001$ \\
\hline
\end{tabular}

llan las poblaciones (Clarke, 1992). Este hecho supone la existencia de pequeñas diferencias latitudinales en la talla de primera madurez de cada especie, y por consiguiente un atraso en el ingreso a la pubertad de las poblaciones que se desarrollan a bajas temperaturas comparado con aquellas que provienen de zonas más cálidas (Thorson, 1936). En Chile, esta tendencia también ha sido confirmada por Hernáez (2001), quien analizando algunas características reproductivas de $P$. granulosus, observó un aumento latitudinal en la talla de primera madurez en diferentes poblaciones de la zona norte.

Al comparar estos resultados con los datos entregados por Hernáez \& Pinheiro (2001) para $P$. granulosus (7,0 $\mathrm{mm} \mathrm{Lc})$, A. angulosus $(10,0 \mathrm{~mm}$ Lc) y A. spinifrons (12,3 mm Lc) capturados en la zona de Arica (18 $30^{\prime} \mathrm{S}$ ), se puede observar el aumento en la talla de madurez en dos de las especies analizadas. Esta comparación confirma lo señalado anteriormente, respecto a la influencia de la latitud sobre la biología de los organismos marinos, hecho de especial relevancia en el manejo pesquero, que en muchas ocasiones hace caso omiso de la proveniencia geográfica de los recursos.

La fecundidad varió de una especie a otra (Tabla 1). En la mayoría de ellas, el efecto del estado de desarrollo sobre el número de huevos no fue significativo y la pérdida de huevos entre estados fue 
despreciable. En vista, que las especies analizadas presentaron fecundidades relativamente bajas (115 a 612 huevos) en comparación con otros crustáceos decápodos (Palma y Arana, 1997), no se consideró necesario transformar los datos a escala logarítmica (Somers, 1991). Sin embargo, es probable que esta operación se requiera en especies con fecundidades mayores, pues el error estadístico aumenta signifi- cativamente en las tallas superiores, cuando la dispersión en la cantidad de huevos portados es mayor (Pinheiro \& Fransozo, 1998).

En los crustáceos, la cantidad de huevos portados está limitada por el espacio disponible bajo el abdomen, por lo tanto las hembras contienen una determinada cantidad de embriones según la forma y ta-

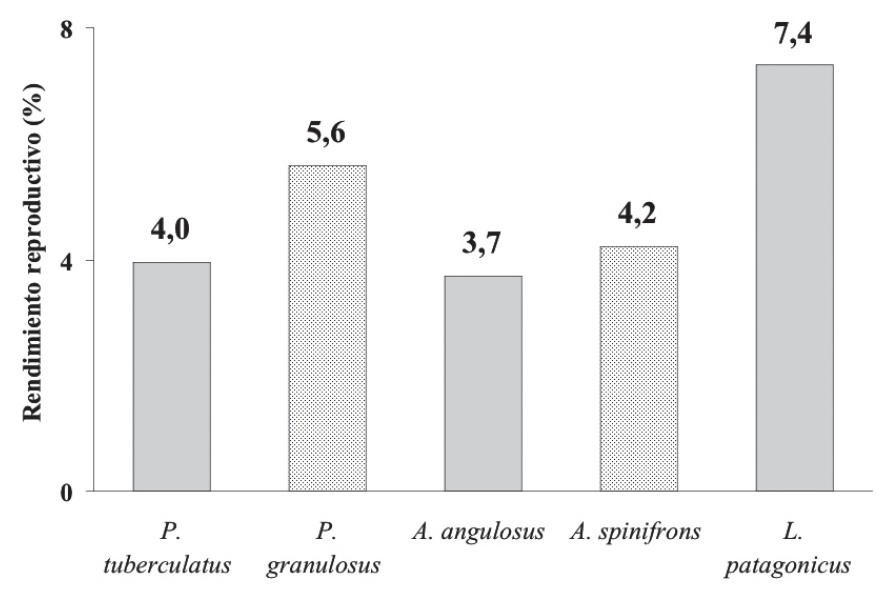

Figura 3. Rendimiento reproductivo (Ro) de las hembras con huevos en estado inicial de desarrollo (E-I), de cinco especies de porcelánidos.

Figure 3. Reproductive output (Ro) of females with eggs in the first stage of development (E-I) in five species of porcellanids.

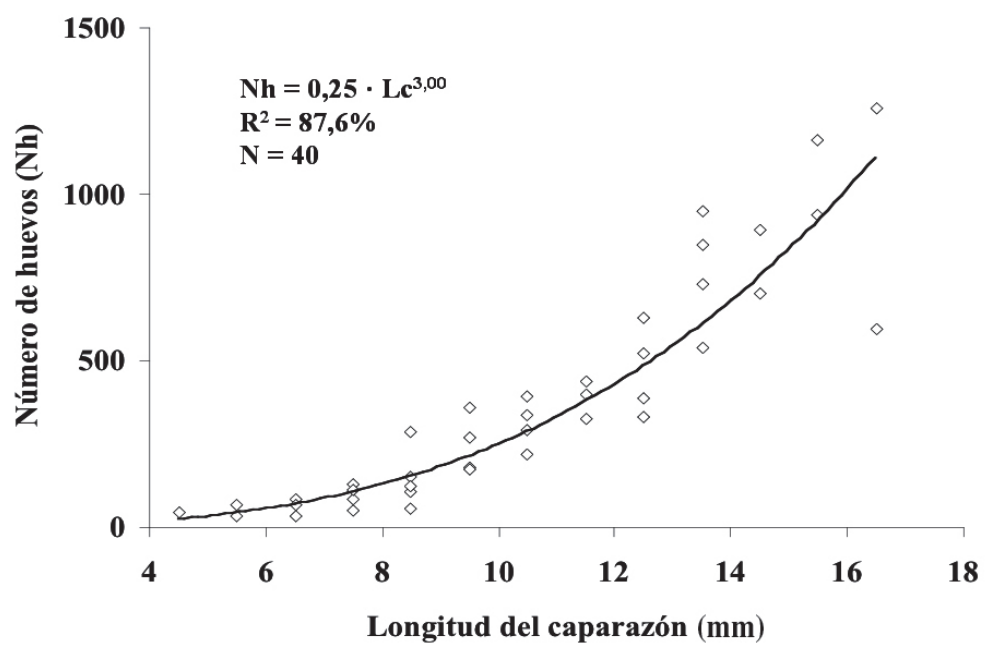

Figura 4. Relación entre el número de huevos portados y la longitud del caparazón por clases de talla del total de hembras analizadas.

Figure 4. Relationships between the number of eggs and the carapace length by size class for the total of females analyzed. 
Tabla 6. Rangos del número de huevos portados y del tamaño de las hembras (Lc) en diferentes especies de crustáceos porcelánidos colectados en la costa chilena.

Table 6. Range of the number of eggs and the female size (Lc) in porcellanid crabs collected along the Chilean coast.

\begin{tabular}{|lrrl|}
\hline Especie & $\begin{array}{c}\text { Rango } \\
\text { número huevos }\end{array}$ & $\begin{array}{c}\text { Rango } \\
\text { Lc (mm) }\end{array}$ & Autor \\
\hline Allopetrolisthes angulosus (Tres Islas) & $6-1.301$ & $6,0-16,8$ & Presente estudio \\
Allopetrolisthes angulosus (Arica) & $8-1.153$ & $6,5-13,1$ & Hernáez \& Pinheiro (2001) \\
Allopetrolisthes angulosus (Valparaíso) & $18-2.582$ & $5,4-15,4$ & Antezana et al. (1965) \\
Allopetrolisthes punctatus (Valparaíso) & $540-16.380$ & $21,9-39,4$ & Antezana et al. (1965) \\
Allopetrolisthes spinifrons (Arica) & $83-1.527$ & $10,8-15,8$ & Hernáez \& Pinheiro (2001) \\
Allopetrolisthes spinifrons (Tres Islas) & $33-2.138$ & $7,6-16,3$ & Presente estudio \\
Allopetrolisthes spinifrons (Valparaíso) & $121-4.548$ & $6,6-16,4$ & Antezana et al. (1965) \\
Liopetrolisthes mitra (Valparaíso) & $62-543$ & $5,0-9,9$ & Antezana et al. (1965) \\
Liopetrolisthes patagonicus (Tres Islas) & $34-1.247$ & $7,0-15,0$ & Presente estudio \\
Pachycheles grossimanus (Valparaíso) & 5.968 & $12,3-20,5$ & Antezana et al. (1965) \\
Petrolisthes granulosus (Arica) & $14-237$ & $5,1-9,0$ & Hernáez \& Pinheiro (2001) \\
Petrolisthes granulosus (Tres Islas) & $40-225$ & $4,7-9,0$ & Presente estudio \\
Petrolisthes granulosus (Taltal) & $35-393$ & $5,3-11,1$ & Hernáez (2001) \\
Petrolisthes laevigatus (Valparaíso) & $162-2.185$ & $8,8-20,0$ & Antezana et al. (1965) \\
Petrolisthes laevigatus (Valdivia) & $56-1141$ & $8,5-18,2$ & Lardies \& Wehrtmann (1996) \\
Petrolisthes tuberculatus (Tres Islas) & $27-537$ & $8,8-13,5$ & Presente estudio \\
Petrolisthes tuberculatus (Valparaíso) & $85-3.683$ & $6,4-18,4$ & Antezana et al. (1965) \\
& & & \\
\hline
\end{tabular}

maño de los huevos, y su tamaño corporal (Reid \& Corey, 1991). Los datos agrupados por rango de talla, muestran que los ejemplares de mayor tamaño aportan mayor cantidad de huevos que los individuos más pequeños (Tabla 2), lo cual ha sido observado en numerosos crustáceos decápodos (Antezana et al., 1965; Jones \& Simons, 1983; Reid \& Corey, 1991; Palma \& Arana, 1997) y específicamente, en varios porcelánidos de la costa chilena (Tabla 6). Consecuentemente, el ajuste de los puntos medios de cada rango y su respectivo número de huevos presentó una alta correlación $(\mathrm{R}=0,94 ; \mathrm{p}<0,001 ; \mathrm{F}$ $=267,67 ; \mathrm{N}=40$, Fig. 4), lo que demuestra la influencia del tamaño de la hembra en su capacidad de portación.

En los crustáceos la fecundidad ha sido representada mediante una serie de modelos matemáticos y en varios trabajos se ha estimado como una función lineal de alguna variable corporal (Antezana et al., 1965; Sanhueza et al., 1975; Lardies \& Wehrtmann, 1996). Sin embargo, es necesario considerar que en la mayoría de los crustáceos decápodos el crecimiento es discontinuo, pues ocu- rre a través de mudas y además, el huevo constituye una medida volumétrica que tiende a aumentar exponencialmente con el tamaño de la hembra (Somers, 1991), lo cual produce una subestimación del número de huevos en las hembras más pequeñas o más grandes (Corey, 1981; Somers, 1991). Por esta razón, muchos investigadores han utilizado el modelo potencial para describir la fecundidad (Hines, 1988; 1991; Reid \& Corey, 1991; Pinheiro \& Fransozo, 1995; Palma \& Arana, 1997), que representa mejor el cambio proporcional de la fecundidad con respecto a la talla corporal (Bagenal, 1967).

La ecuación $\mathrm{Nh}=0,25 \cdot \mathrm{Lc}^{3,0}$ estimada para el total de las hembras mostró un buen ajuste de los puntos medios y explicó el $87,6 \%$ de la variación total del número de huevos en función de la talla corporal. Además, la pendiente basada en el modelo alométrico es igual a 3 lo cual según Somers (1991), constituye una buena prueba de que en este modelo, el número de huevos está bien representado (Fig. 4). La varianza explicada fue similar a la determinada para el langostino colorado $(88,5 \%)$ en la zona 
centro-sur de Chile (Palma \& Arana, 1997). Comparativamente con el ajuste de los datos empíricos, la relación entre los puntos medios, mostró un importante aumento en la confiabilidad del modelo (Fig. 1, $\mathrm{R}^{2}=59,2$; Fig. 3, $\mathrm{R}^{2}=87,6$ ). Es probable que este procedimiento minimice el error producido por la variabilidad de los datos y por el aumento de la dispersión hacia los individuos de tallas mayores.

El tamaño y volumen del huevo, así como el tiempo de incubación, son fundamentales para establecer la estrategia reproductiva de una especie determinada (McEdward, 1997). En este sentido, se presume que el tiempo de incubación está directamente relacionado con el tamaño del huevo (Levitan, 1996). Esto permite suponer que los embriones de mayor tamaño deberían demorar menos tiempo en eclosionar que los más pequeños. Sin embargo, la mayor parte de los estudios realizados describen el desarrollo larval sin considerar el tiempo de incubación y el volumen del huevo, lo cual impide establecer el tipo de estrategia que las hembras adoptan durante un periodo reproductivo determinado.

Según Lardies \& Wehrtmann (1996), el crecimiento del embrión durante el desarrollo está limitado por el grosor y resistencia de la membrana, lo que puede ser importante en aquellos porcelánidos cuyas poblaciones se encuentran sometidas a mayor presión de desecación durante los periodos de baja marea. Los resultados obtenidos indican una tendencia inversa entre el patrón de zonación de cada especie y el aumento de volumen entre los estados de desarrollo, confirmando así que la membrana del huevo puede ser uno de los factores limitantes en el crecimiento del embrión. En comparación con otros decápodos como calápidos, cáncridos y xántidos, los porcelánidos poseen huevos relativamente grandes, con membranas gruesas y altamente resistentes a la desecación. A pesar que el volumen promedio en $A$. angulosus y A. spinifrons fue superior al registrado por Hernáez \& Pinheiro (2001), es probable que al interior de la misma especie, la diferencia en el tamaño del embrión en poblaciones de áreas geográficas distintas, puede ser una respuesta reproductiva de las hembras a condiciones propias del ambiente y no a una tendencia latitudinal (Hernáez, 2001). La alta correlación entre el volumen promedio y el contenido de peso seco del huevo en cada especie, demuestra que los huevos de mayor tamaño son más ricos en contenido, lo que tiene relación con la energía dispuesta para el crecimiento del embrión (Clarke, 1992).
La inversión energética de las hembras en el proceso reproductivo constituye uno de los aspectos más interesantes en la biología reproductiva de los crustáceos. Esta variable ha sido estimada mediante el rendimiento reproductivo (Ro) de las hembras (Havenhead \& Todd, 1989; Clarke et al., 1991). Los valores obtenidos para los porcelánidos de 3,7-7,4\%, indican que están dentro del rango de 3-22\% del peso seco de la hembra dado para algunos braquiuros (Hines, 1991). Los datos reportados por Lardies \& Wehrtmann (1996) y Hernáez \& Pinheiro (2001) para porcelánidos de otras regiones de la costa chilena muestran, en general, valores de Ro diferentes a los registrados en Tres Islas. Es posible que las condiciones del medio también influyan en el comportamiento de esta variable y se requiera de otro tipo de estudios para establecer mejores comparaciones entre poblaciones distantes geográficamente.

La energía que las hembras invierten en la reproducción debería presentar alguna tendencia asociada a la edad o tamaño de los individuos. Sin embargo, los resultados indicaron que el Ro de cada especie, no se correlacionó positivamente con el tamaño de las hembras $(\mathrm{R}=0,06 ; \mathrm{p}<0,001 ; \mathrm{F}=0,57$; $\mathrm{N}=129$ ), lo cual se repitió al comparar el Ro promedio entre cada especie (Fig. 3). Sumado a esto, las hembras no invierten más energía en la reproducción a medida que crecen, puesto que el Ro estimado para de las tallas mayores es inferior al calculado para los ejemplares mas pequeños de cada especie. Estos resultados sugieren una discusión más profunda sobre la eficacia de este método como medida de la inversión reproductiva en los crustáceos marinos y se estima necesario contar con un buen indicador de la inversión reproductiva que sea coherente con la historia de vida de estos organismos.

\section{REFERENCIAS}

Annala, J.H., J.L. Mckoy, J.D. Booth \& R.B. Pike. 1980. Size at the onset of sexual maturity in female Jasus edwarsii (Decapoda, Palinuridae) in New Zealand. N.Z.J. Mar. Freshwater Res., 14: 217-228.

Antezana, T., E. Fagetti \& M.T. López. 1965. Observaciones bioecológicas en decápodos de Valparaíso. Rev. Biol. Mar., Valparaíso, 12: 1-60.

Bagenal, T.B. 1967. A short review of fish fecundity. En: S.D. Gerking (ed.). The biological basis of 
freshwater fish production. Blackwell, Edinburgh, pp. 89-111.

Caddy, J.F. 1989. Marine invertebrate fisheries. Their assessment and management. John Wiley \& Sons, New York, 752 pp.

Carvacho, A. 1980. Los porcelánidos del Pacífico americano: un análisis biogeográfico (Crustacea, Decapoda). An. Centro Cienc. Mar. Limnol., Univ. Nac. Autón. México, 7(2): 249-258.

Clarke, A. 1992. Reproduction in the cold: Thorson revisited. Invertebr. Reprod. Dev., 22(1-3): 175184.

Clarke, A., C.C. Hopkins \& E.M. Nilssen. 1991. Egg size and reproductive output in the deep-water prawn Pandalus borealis Kroyer, 1838. Funct. Ecol., 5: 724-730.

Corey, S. 1981. Comparative fecundity and reproductive strategies in seventeen species of the Cumacea (Crustacea, Peracarida). Mar. Biol., 62: 65-72.

Corey, S. \& D.M. Reid. 1991. Comparative fecundity of decapod crustaceans. I. The fecundity of thirty tree species of nine families of caridean shrimps. Crustaceana, 60(3): 270-294.

Díaz, H. 1980. The mole crab Emerita talpoida (Say): a case of changing life history pattern. Ecol. Monogr., 50: 437-456.

Guzmán, G. 1999. Clave para los crustáceos decápodos anomuros marinos de Chile. Ediciones Campus. Universidad Arturo Prat, Iquique, 52 pp.

Haig, J. 1955. The Crustacea Anomura of Chile. Reports of the Lund University Chile Expedition 1948-1949. Lunds Univ. Arsskrift, 51(12): 1-68.

Haig, J. 1960. The Porcellanidae (Crustacea Anomura) of the Eastern Pacific. Allan Hancock Pacific Exped., 24: 1-440.

Havenhead, J.N. \& C.D. Todd. 1989. Reproductive effort of the nudibranch mollusc Adalaria proxima (Alder and Hancock) and Onchidoris muricata (Muller). An evaluation of techniques. Func. Ecol., 3: 153-163.

Hernáez, P. 2001. Producción y rendimiento reproductivo en Petrolisthes granulosus (Decapoda, Anomura, Porcellanidae) en diferentes localidades del norte de Chile: una comparación latitudinal. Invest. Mar., Valparaíso, 29(1): 73-81.
Hernáez, P. \& M.A. Pinheiro. 2001. Production and reproductive output of four porcelain crab species from northern Chile. Nauplius, 9(1): 43-52.

Herring, P.J. 1974. Size, density and lipid content of some decapod eggs. Deep-Sea Res., 21: 91-94.

Hines, A.H. 1988. Fecundity and reproductive output in two species of deep-sea crabs, Geryon fenneri and G. quinquedens (Decapoda: Brachyura,). J. Crust. Biol., 8: 557-562.

Hines, A.H. 1991. Fecundity and reproductive output in nine species of Cancer crabs (Crustacea, Brachyura, Cancridae). Can. J. Fish. Aquat. Sci., 48: 267-275.

Jones, M.B. \& M.J. Simons. 1983. Latitudinal variation in reproductive characteristics of a mud crab, Helice crassa (Grapsidae). Bull. Mar. Sci., 33: $656-670$.

Kinne, O. 1970. Temperature: animals-invertebrates. En: O. Kinne (ed.). Marine Ecology. I. Environmental factors. Wiley-Interscience, London, pp. 407-514.

Lardies, M.A. \& I.S. Wehrtmann. 1996. Aspects of the reproductive biology of Petrolisthes laevigatus (Guérin, 1835) (Decapoda, Anomura, Porcellanidae). I. Reproductive output and chemical composition of eggs during embryonic development. Arch. Fish. Mar. Res., 43(2): 121135 .

Levitan, D. 1996. Predicting optimal and unique egg free-spawning marine invertebrates. Amer. Natural., 148(1): 174-188.

McEdward, L.R. 1997. Reproductive strategies of marine benthic invertebrates revisited: facultative feeding by planktotrophic larvae. Amer. Natur., 150(1): 48-72.

Palma, S. 1980. Larvas de crustáceos decápodos capturados frente a la costa de Valparaíso. Invest. Mar., Valparaíso, 8: 129-144.

Palma, S. \& P. Arana. 1997. Aspectos reproductivos del langostino colorado (Pleuroncodes monodon H. Milne Edwards, 1837) frente a la costa de Concepción, Chile. Invest. Mar., Valparaíso, 25: $203-$ 221.

Pinheiro, M.A. \& A. Fransozo. 1995. Fecundidade de Pachycheles haigae Rodríguez Da Costa, 1960 (Crustacea, Anomura, Porcellanidae) em Ubatuba 
(SP), Brasil. Rev. Brasileña Biol., 55(4): 623-631.

Pinheiro, M.A. \& A. Fransozo. 1998. Sexual maturity of the speckled swimming crab Arenaeus cribrarius (Lamarck, 1818) (Decapoda: Brachyura: Portunidae), in the Ubatuba littoral, São Paulo State, Brazil. Crustaceana, 71(4): 434-452.

Reid, D.M. \& S. Corey. 1991. Comparative fecundity of decapod crustaceans. II. The fecundity of fifteen species of anomuran and brachyuran crabs. Crustaceana, 61(2): 175-189.

Sanhueza, E., N. Bahamonde \& M.T. López. 1975. Petrolisthes granulosus (Guérin) en biocenosis supramareales de El Tabo (Crustacea, Decapoda, Anomura). Bol. Mus. Nac. Hist. Nat., 34: 121-136.

Somers, K.M. 1991. Characterizing size-specific fecundity in crustaceans. En: F.R. Schram (ed.). Crustacean egg production. A.A. Balkema, Rotterdam. Crustacean Issues, 7: 357-378.

Sokal, R. \& F. Rohlf. 1981. Biometry. The principles and practice of statistics in biological research. Freeman, New York, 859 pp.

Stechey, D. \& K. Somers. 1995. Potential, realized, and actual fecundity in the crayfish Orconectes imunis from southwestern Ontario. Can. J. Zool., 73: 672-677.

Recibido: 27 mayo 2003; Aceptado: 10 octubre 2003
Swartz, R.C. 1978. Reproductive and molt cycles in the xanthid crab Neopanope sayi. Crustaceana, 34: 15-32.

Thorson, G. 1936. The larval development, growth and metabolism of Arctic marine invertebrates, compared with those of the other seas. Meddr. Grønland., 100: 1-155.

Ulloa, R. \& S. Palma. 1998. Distribución larval de Petrolisthes violaceus, $P$. laevigatus y Allopetrolisthes angulosus en el plancton de la bahía de Valparaíso. Rev. Biol. Mar. Oceanogr., 33(1): 125-138.

Viviani, C. 1969. Los Porcellanidae (Crustacea, Anomura) chilenos. Beit. Z. Neotrop. Fauna, 4(1): 40-56.

Wenner, A.M., C. Fusaro \& A. Oaten. 1974. Size at onset of sexual maturity and growth rate in crustacean populations. Can. J. Zool., 52: 10951106.

Werding, B. 1984. Porcelánidos (Crustacea, Anomura, Porcellanidae) de la Isla de Providencia, Colombia. An. Inst. Invest. Mar., 14: 3-16.

Werthmann, I.S. \& G. Andrade. 1998. Egg production in Heterocarpus reedi from northern Chile, with a comparison between iced and living females. Ophelia, 49(1): 71-82. 